\title{
Longitudinal medical resources and costs among type 2 diabetes patients participating in the Trial Evaluating Cardiovascular Outcomes with Sitagliptin (TECOS)
}

\author{
Shelby D. Reed PhD ${ }^{1}$ (1) | Yanhong Li MS ${ }^{1}$ | Jose Leal PhD ${ }^{2}$ | Larry Radican PhD ${ }^{3}$ | \\ Amanda I. Adler $\mathrm{MD}^{4}$ | Joakim Alfredsson $\mathrm{MD}^{5}$ | John B. Buse $\mathrm{MD}^{6}$ | \\ Jennifer B. Green $M^{1}$ | Keith D. Kaufman $M^{3} D^{3}$ Axel Riefflin $M^{7}$ | \\ Frans Van de Werf $\mathrm{MD}^{8} \mid$ Eric D. Peterson $\mathrm{MD}^{1} \mid$ Alastair M. Gray $\mathrm{PhD}^{2 \dagger}$ | \\ Rury R. Holman $\mathrm{MB} \mathrm{ChB}^{2 \dagger}$ (1) | for the TECOS Study Group
}

${ }^{1}$ Duke Clinical Research Institute, Duke University School of Medicine, Durham, North Carolina

${ }^{2}$ Diabetes Trials Unit, Oxford Centre for Diabetes, Endocrinology and Metabolism, University of Oxford, Oxford, UK

${ }^{3}$ Merck \& Co. Inc., Kenilworth, New Jersey

${ }^{4}$ Institute of Metabolic Science, Addenbrooke's Hospital, Cambridge, UK

${ }^{5}$ Linköping University Hospital, Linköping, Sweden

${ }^{6}$ Division of Endocrinology, University of North Carolina, Chapel Hill, North Carolina

${ }^{7} \mathrm{GMP}$, Husby, Germany

${ }^{8}$ Department of Cardiovascular Medicine, University Hospitals, Leuven, Belgium

\section{Correspondence}

Shelby D. Reed, PhD, Duke Clinical Research Institute, PO Box 17969, Durham, NC 27715.

Email: shelby.reed@duke.edu

Funding information

This study was funded by Merck Sharp \& Dohme Corp., a subsidiary of Merck \& Co., Inc., Kenilworth, New Jersey.

\begin{abstract}
Aims: TECOS, a cardiovascular safety trial (ClinicalTrials.gov identifier: NCT00790205) involving 14671 patients with type 2 diabetes and cardiovascular disease, demonstrated that sitagliptin was non-inferior to placebo for the primary composite cardiovascular outcome when added to best usual care. This study tested hypotheses that medical resource use and costs differed between these 2 treatment strategies.

Materials and methods: Information concerning medical resource use was collected on case report forms throughout the trial and was valued using US costs for: Medicare payments for hospitalizations, medical procedures and outpatient visits, and wholesale acquisition costs (WAC) for diabetes-related medications. Hierarchical generalized linear models were used to compare resource use and US costs, accounting for variable intercountry practice patterns. Sensitivity analyses included resource valuation using English costs for a UK perspective.

Results: There were no significant differences in hospitalizations, inpatient days, medical procedures, or outpatient visits during follow-up (mean and median 3.0 years in both groups). Hospitalization rates appeared to diverge after 2 years, with lower rates among sitagliptin-treated vs placebo patients after 2.5 years (relative rate, 0.90 [95\% Cl, 0.83-0.97]; $P=.01$ ). Mean medical costs, exclusive of study medication, were 11937 USD in the sitagliptin arm and 12409 USD in the placebo arm $(P=.06)$. Mean sitagliptin costs based on undiscounted WAC were 9978 USD per patient. Differential UK total costs including study drug costs were smaller (911 GBP), primarily because of lower mean costs for sitagliptin (1072 GBP).

Conclusions: Lower hospitalization rates across time with sitagliptin slightly offset sitagliptin treatment costs over 3 years in type 2 diabetes patients at high risk for cardiovascular events.
\end{abstract}

KEYWORDS

costs, cost analysis, diabetes, dipeptidyl peptidase- 4 inhibitor, sitagliptin

\section{1 | INTRODUCTION}

The economic implications of type 2 diabetes are immense. Disability-adjusted life-years associated with diabetes increased

These authors contributed equally. globally by $29 \%$ between 2005 and $2015 .{ }^{1}$ Estimated global health expenditures for diabetes ranged from 612 billion USD to 1.10 trillion USD per year in 2014. ${ }^{2}$ These extraordinary numbers are expected to grow as the number of individuals with diabetes worldwide is projected to rise from 415 million in 2015 to 642 million by $2040 .^{3}$ 
Patients with type 2 diabetes have rates of coronary heart disease and ischemic stroke that are 2-fold higher than those of patients without type 2 diabetes, even after adjusting for traditional risk factors. ${ }^{4}$ Although there is evidence of long-term declines in the excess risk of death and cardiovascular outcomes in individuals with type 2 diabetes, ${ }^{5}$ death rates with vascular causes and non-vascular causes, including cancer and infectious diseases, remain significantly higher among individuals with type 2 diabetes. ${ }^{6}$ Numerous therapeutic strategies are available to improve glycaemic control, with the goal of reducing morbidity and mortality, but questions about potential adverse effects on major cardiovascular events have been raised, ${ }^{7,8}$ leading regulators to require large-scale cardiovascular safety trials of new treatments for type 2 diabetes. ${ }^{9,10}$ These trials typically strive for equivalent levels of glycaemic control between study arms to isolate the potential direct impact of the study drug on major adverse cardiovascular events from possible indirect effects attributable to better glycaemic control.

The Trial Evaluating Cardiovascular Outcomes with Sitagliptin (TECOS), a placebo-controlled cardiovascular safety study, was designed to test the impact of adding sitagliptin, a dipeptidyl peptidase-4 (DPP-4) inhibitor, to usual care in patients with type 2 diabetes and established cardiovascular disease who did not receive other DPP-4 inhibitors or glucagon-like peptide-1 (GLP-1) receptor agonists. ${ }^{11}$ A prespecified secondary objective was to compare medical resource use during the trial by treatment arm. As an extension, we valued all medical resources using US cost weights and compared direct medical costs between study arms. As a sensitivity analysis, we also valued medical resources using UK cost weights, to evaluate the results in a different setting.

\section{2 | RESEARCH DESIGN AND METHODS}

\section{1 | TECOS trial design and results}

Eligible patients had a history of cardiovascular disease. Baseline glycated haemoglobin ( $\mathrm{HbA1c}$ ) range for enrollment was $6.5 \%$ to $8.0 \%$ $(48-64 \mathrm{mmol} / \mathrm{mol})$. Patients were randomized to either placebo or sitagliptin (100 or $50 \mathrm{mg}$ daily, depending on renal function). The primary endpoint was time to first cardiovascular-related death, nonfatal myocardial infarction, non-fatal stroke or hospitalization for unstable angina. The study was powered to demonstrate noninferiority of sitagliptin added to usual care vs usual care alone, with $90 \%$ power to exclude a $30 \%$ increased risk of cardiovascular events with sitagliptin-based care. ${ }^{11}$ The protocol for the study was approved by the ethics committees associated with all participating trial sites, and all participants provided written informed consent.

In TECOS, the intention-to-treat analysis population included 14671 randomized participants from 38 countries. Participants in both study groups were followed for a mean of 3.0 years (sitagliptin, 3.01 [SD, 0.96]; placebo, 2.99 [SD, 0.97]). ${ }^{12}$ Approximately onequarter of participants in both arms discontinued study medication prior to the end of follow-up (sitagliptin, 26.1\%; placebo, 27.5\%). Despite the aim of equal glycaemic control between groups, mean HbA1c levels were, on average, 0.3\% lower with sitagliptin (95\% confidence interval $[\mathrm{Cl}],-0.32$ to -0.27$)$. Sitagliptin was shown to be non-inferior to placebo for the primary composite cardiovascular outcome (hazard ratio [HR], 0.98; 95\% Cl, 0.88-1.09; $P$ for non-inferiority $<.001$ ). All-cause mortality was similar in both arms (HR, 1.01; $95 \%$ $\mathrm{Cl}, 0.90-1.14)$. The proportion of patients initiating insulin during the trial was significantly lower in the sitagliptin group (9.7\%) compared to the placebo group (13.2\%; $P<.001$ ). Severe hypoglycaemia occurred in $2.0 \%$ of patients randomized to sitagliptin compared with $1.7 \%$ of patients randomized to placebo $(\mathrm{HR}, 1.13 ; 95 \% \mathrm{Cl}$, $0.89-1.44)$

\section{2 | Economic evaluation}

Academic health economics teams from the Duke Clinical Research Institute and the University of Oxford Health Economics Research Centre led and performed the economic evaluation. An analysis plan, finalized on August 7, 2015, was used to guide cost assignment and planned statistical analysis.

\section{3 | Medical resource use and cost assignment}

Data on medical resource use, including hospitalizations, cardiovascular procedures, study visits, outpatient visits to usual diabetes care providers or other providers, concomitant medications, and study drug were collected at 4, 8, and 12 months, and then every 6 months through to study end.

For each hospitalization, admission and discharge dates were recorded, along with the primary discharge diagnosis documented as 1 of 32 prespecified diagnoses or free text. All major cardiovascular and renal procedures were recorded, as were specific procedures for management of congestive heart failure, cardiac ischaemic event, stroke/transient ischaemic attack and pancreatitis.

Numbers of treatment days on study drugs were derived from trial records, accounting for intermittent discontinuations. Information for other diabetes medicines included drug name and daily dose, but start and stop dates were not collected, hence drug use recorded as "yes" was assumed to continue until "no" was recorded at a subsequent visit.

\section{4 | Cost assignment}

Costs were assigned to medical resource use reported for all patients in the trial. US hospital cost assignment was based on Medicare reimbursement rates for corresponding Diagnosis-Related Group (DRG) codes assigned to prespecified discharge diagnoses and procedures (Tables S1 and S2 in File S1) and high-frequency free-text discharge diagnoses recorded in TECOS. To account for systematic differences in length of stay across patients in different countries with the same diagnosis, we applied an approach to inpatient cost assignment used in another large multinational trial. ${ }^{13}$ For each DRG code, cost per inpatient day was estimated by dividing the 2015 Medicare reimbursement by the median length of stay published by the Centers for Medicare and Medicaid Services, ${ }^{14}$ then merged with hospitalizations using the assigned DRG code. We then adjusted lengths of stay recorded for hospitalizations outside the USA to approximate the 
length of stay had the patient been treated at a US facility. This was done by calculating the ratio of mean length of stay across all hospitalizations in the USA to the mean length of stay in each non-US country participating in TECOS. The resulting country-specific ratio was multiplied by the length of stay for each hospitalization record and the DRG-specific daily inpatient cost.

The 2015 Medicare Physician Fee Schedule was used to estimate costs for physician services using Current Procedural Terminology codes (Tables S3 and S4 in File S1) ${ }^{15}$ Daily costs for concomitant diabetes medications were provided by Merck \& Co., Inc., based on 2015 wholesale acquisition costs (WAC) published by First Databank, which represent the manufacturer's published list price to wholesalers and may not represent actual transactional prices. ${ }^{16}$ As generic drugs were available from multiple manufacturers, the lowest and highest costs for specific daily doses of concomitant diabetes drugs were developed. Lowest costs for generic drugs were used in the basecase analysis. The daily cost assigned for sitagliptin was 11.02 USD across all doses. All costs incurred beyond the first year were discounted at $3 \%$ per year.

\section{5 | Statistical analysis}

Descriptive statistics, including proportions, means, standard deviations and medians, summarized counts of medical resource use and costs by treatment group. Mean cumulative counts of hospitalizations per patient that account for censoring across time were plotted, ${ }^{17}$ and annual hospitalization rates were computed by dividing the total number of hospitalizations for each treatment group in each year by the total duration of follow-up in the corresponding year.

To account for the nested structure of the multinational trial data, generalized linear mixed models were used to compare counts of medical resource use between treatment groups using SAS's PROC GLIMMIX with Laplace estimation (SAS Institute, Version 9.4). The models included treatment assignment as the independent variable, with the log of each participant's duration of follow-up as an offset variable to adjust for differences in observation time. Treatment assignment was modeled as a fixed effect and countries

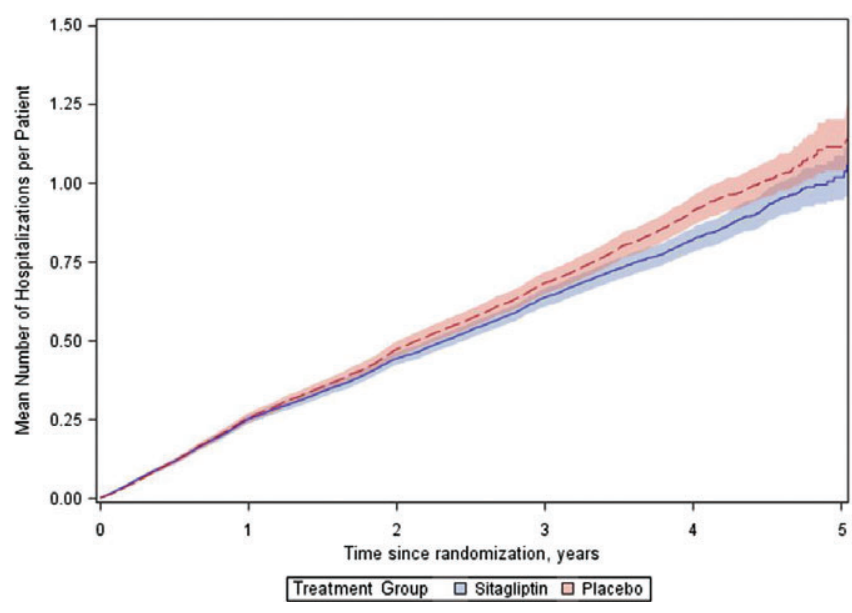

FIGURE 1 Mean cumulative number of hospitalizations per patient and $95 \%$ confidence intervals by treatment group (see Table 3 for results by minimum follow-up duration) were modeled as random intercepts, to allow rates of medical resource use and costs in the placebo group to vary across countries while modeling the relative impact of sitagliptin as a fixed effect across countries. To compare medical resource use counts (hospitalizations, inpatient days and outpatient visits), the models were specified with negative binomial error distributions and log links. Models to compare diabetes drug-days relied on normal distributions and identity links. For cost comparisons, gamma error distributions and log links were used. The exponentiated parameter estimates from these models represent relative rates (RR) for resource use and means ratios for costs. To provide additional descriptive information about treatment effects on resource use and costs on an absolute scale, differences in mean counts were computed and corresponding $95 \% \mathrm{Cls}$ were estimated using the bias-corrected percentile method based on 1000 non-parametric bootstrap replications. ${ }^{18}$

\section{6 | Sensitivity analysis}

Several sensitivity analyses were performed to evaluate the impact of alternative methods and assumptions on study findings. First, in lieu of assigning the lowest WACs reported for generic diabetes medications, the highest WACs were assigned. Second, when adjusting lengths of stay for hospitalizations outside the USA, we calculated the conversion ratios using median rather than mean lengths of stay. Third, we replicated the entire analysis using costs from the UK rather than the USA (see Suporting Information Methods for details).

\section{7 | Subgroup analysis}

Subgroup analyses were performed to determine whether adding sitagliptin to usual care had a differential impact on all-cause hospitalizations. Selection of subgroups mirrored those that were prespecified or conducted for the primary clinical endpoint. ${ }^{12}$

\section{8 | Post-hoc analysis}

Post-hoc analyses were performed to compare rates of hospitalizations between groups among patients with longer periods of follow-up.

\section{3 | RESULTS}

Participants randomized to receive sitagliptin in addition to usual care were hospitalized 4803 times compared with 5168 times among participants randomized to receive placebo (Figure 1), representing $34.1 \%$ and $34.6 \%$ of participants, respectively $(P=.26)$, being hospitalized at least once over the 3 -year follow-up period. The mean number of hospitalizations was 0.66 in the sitagliptin arm and 0.70 in the placebo arm (RR, $0.95[95 \% \mathrm{Cl}, 0.90-1.02] P=.14)$ (Figure 1). Mean number of inpatient days was 5.50 days for the sitagliptin group and 5.74 days for the placebo group $(P=.94)$ (Table 1). Mean count of outpatient visits was 19.4 per participant in both groups $(P=.85)$. Mean number of concomitant diabetes drug-days was 1635 
and 1673 per participant for the sitagliptin and placebo groups, respectively, or approximately 1.5 diabetes drugs per day per participant $(P=.06)$. When including sitagliptin, diabetes drug-days averaged 2.3 per day per participant among those randomized to the sitagliptin arm. Mean number of days on insulin therapy was 234 per participant in the sitagliptin arm compared to 241 days in the placebo $\operatorname{arm}(P=.26)$.

Inpatient costs over the follow-up period averaged 6947 USD in the sitagliptin arm and 7377 USD in the placebo arm (Table 1). Outpatient care costs were approximately 1465 USD in both study groups. Mean total costs for medical resources, exclusive of sitagliptin, were 11937 USD in the sitagliptin arm and 12409 USD in the placebo arm. Mean costs for sitagliptin were estimated at 9978 USD, resulting in total within-trial costs for the sitagliptin group of 21915 USD.

\section{1 | Sensitivity analysis}

When concomitant diabetes medicines were assigned the upper (rather than lower) bound of prices across generic manufacturers, diabetes medications costs averaged 4126 USD in the sitagliptin arm vs 4198 USD in the placebo arm, a difference of 72 USD. Using lengthof-stay conversion ratios based on median (rather than mean) length of stay in the USA relative to other countries, estimated inpatient costs were 901 USD higher in the sitagliptin arm and 931 USD higher in the placebo arm. And, when resource use was valued using UK costs, inpatient costs over the follow-up period averaged 2629 GBP in the sitagliptin arm and $2760 \mathrm{GBP}$ in the placebo arm (Table 1). Outpatient care costs were 2648 GBP in the sitagliptin arm and 2654 GBP in the placebo arm. Mean total costs for medical resources, exclusive of sitagliptin, were $£ 6058$ in the sitagliptin arm and 6219 GBP in the placebo arm. Mean costs for sitagliptin were estimated at 1072 GBP, resulting in total within-trial costs for the sitagliptin arm of $7130 \mathrm{GBP}$, which was $911 \mathrm{GBP}$ higher than those for the placebo $\operatorname{arm}(P<.001)$.

\section{2 | Subgroup analysis}

Effects of sitagliptin on hospitalizations by subgroup are reported in Table 2. Only 1 interaction term was statistically significant at $P<.05$, suggesting that younger patients ( $<75$ years of age) may experience fewer hospitalizations when treated with sitagliptin. Within subgroups, only $1 P$ value was $<.05$, occurring among patients with diabetes duration of 5 to $<15$ years.

\section{3 | Post-hoc analysis}

Post-hoc analyses revealed that patients with at least 2.5 years of follow-up experienced a $10 \%$ lower rate of hospitalization (ie, RR, $0.90 ; P=.01)$ when treated with sitagliptin compared to placebo (Table 3). This observation was maintained among smaller groups of patients with at least 3.0 and 3.5 years of follow-up.

\section{4 | DISCUSSION}

TECOS randomized 14671 patients across 38 countries to evaluate long-term safety of sitagliptin in patients with type 2 diabetes and pre-existing cardiovascular disease. Clinical findings confirmed that sitagliptin, when added to usual care (but excluding DPP-4 inhibitors and GLP-1 receptor agonists), is non-inferior to placebo plus usual care with regard to its impact on cardiovascular outcomes, including cardiovascular death, non-fatal myocardial infarction, non-fatal stroke, and hospitalization for unstable angina, and had no impact on the incidence of hospitalization for heart failure. ${ }^{19}$

The TECOS trial allowed providers to help enrolled patients achieve individualized glycaemic control goals with their choice of open-label antihyperglycaemic agents, excluding other DPP-4 inhibitors or GLP-1 receptor agonists and, as a randomized and blinded clinical trial, permits an internally valid evaluation of whether the addition of sitagliptin had an independent impact on medical resource use and associated costs. However, it should be noted that not all resources of relevance in type 2 diabetes (such as glucose testing strips) were measured. In addition, despite the aim of achieving glycaemic equipoise, participants randomized to sitagliptin had mean HbA1c values $0.3 \%$ lower, on average, than those of the placebo arm, suggesting possible suboptimal addition of antihyperglycaemic agents in the control arm during the study, and commensurately lower medication costs in that arm than if equal glycaemic control had been achieved. The study design also limited enrollment to participants with $\mathrm{HbA} 1 \mathrm{c}$ levels of $6.5 \%$ to $8.0 \%$ at baseline, potentially restricting the opportunity to demonstrate effects of improved glycaemic control, which may have reduced the generalizability of our findings.

Drug costs, monitoring costs and costs to manage drug-related side effects are essential components of an economic evaluation of sitagliptin. In our resource utilization study, we observed slightly fewer drug treatment days with concomitant diabetes drugs and fewer days on insulin in the sitagliptin arm, but little cost saving because of the low cost of generic diabetes drugs and the modest absolute differences in total insulin use between arms, given the proportion of patients $(23 \%)$ using insulin at study entry. At the outset of the trial, we considered that sitagliptin might make it easier to achieve personalized glucose targets and reduce the number of outpatient visits relative to usual care plus placebo, but in practice, the mean number of outpatient visits reported was nearly identical. However, it is possible that additional care for drug initiation or monitoring occurred during protocol-required visits that was not captured in the context of the TECOS trial, which may have reduced the opportunity to observe additional differences in resource utilization between arms.

The $0.3 \%$ lower $\mathrm{HbA} 1 \mathrm{c}$ values in the sitagliptin arm may have contributed to several indications that the treatment lowered hospitalization rates. The $95 \% \mathrm{Cl}$ corresponding to the difference in mean hospitalizations between treatment arms excluded zero, consistent with a nominally significant finding at $P<.05$, but the $P$ value from the prespecified hierarchical model to compare hospitalization rates in the primary analysis was not statistically significant. We also observed cases where mean costs in the sitagliptin arm were lower 
TABLE 1 Medical resource use and costs throughout trial

\begin{tabular}{|c|c|c|c|c|c|}
\hline & Sitagliptin $(n=7332)$ & Placebo $(n=7339)$ & Difference $(95 \% \mathrm{Cl})^{\mathrm{a}}$ & $\begin{array}{l}\text { Relative rate/means ratio } \\
(95 \% \mathrm{Cl})\end{array}$ & $P$ value \\
\hline \multicolumn{6}{|l|}{ Medical resource use } \\
\hline All-cause hospitalizations & $0.66(1.29)$ & $0.70(1.43)$ & $-0.05(-0.09$ to -0.01$)$ & $0.95(0.90-1.02)$ & .14 \\
\hline Inpatient days & $5.50(16.38)$ & $5.74(16.54)$ & $-0.24(-0.77$ to 0.24$)$ & $1.00(0.90-1.10)$ & .94 \\
\hline Outpatient care visits & $19.42(17.36)$ & $19.43(17.35)$ & $-0.01(-0.52$ to 0.58$)$ & $1.00(0.98-1.02)$ & .85 \\
\hline Diabetes drug-days ${ }^{\mathrm{b}}$ & $1635(833)$ & $1673(861)$ & $-38(-66$ to -11$)$ & $0.99(0.97-1.00)$ & .06 \\
\hline Inpatient care & 6947 (19 935) & $7377(20066)$ & $-430(-1109$ to 168$)$ & $1.08(0.97-1.21)$ & .17 \\
\hline Outpatient care & 1465 (1413) & $1464(1364)$ & 1 (-39 to 50$)$ & $1.00(0.97-1.03)$ & .88 \\
\hline Diabetes medications & $3524(7644)$ & 3567 (7623) & $-43(-269$ to 216$)$ & $0.96(0.91-1.02)$ & .18 \\
\hline Total, excluding sitagliptin & 11937 (22 265) & 12409 (22 283) & -472 (-1193 to 247$)$ & $1.04(1.00-1.09)$ & .06 \\
\hline Sitagliptin & $9978(4527)$ & $0(0)$ & & & \\
\hline Inpatient care & 2629 (19 935) & $2760(20066)$ & $-131(-406$ to 116$)$ & $1.05(0.94-1.17)^{\mathrm{e}}$ & .20 \\
\hline Outpatient care & $2648(2287)$ & $2654(2294)$ & $-6(-73$ to 73$)$ & $1.00(0.97-1.03)$ & .94 \\
\hline Diabetes medications & $781(870)$ & 805 (868) & $-24(-50$ to -5$)$ & $0.99(0.96-1.02)$ & .38 \\
\hline Total, excluding sitagliptin & $6058(8769)$ & $6219(9061)$ & $-161(-445$ to 119$)$ & $1.02(0.99-1.05)$ & .22 \\
\hline Sitagliptin & $1072(485)$ & $0(0)$ & & & \\
\hline Total costs & 7130 (8822) & 6219 (9061) & 911 (627 to 1201$)$ & $1.19(1.16-1.23)$ & $<.0001$ \\
\hline
\end{tabular}

Data are given as mean (SD) unless otherwise indicated.

a 95\% Cls based on bias-adjusted percentile method with non-parametric bootstrapping.

${ }^{b}$ Represents the number of diabetes drugs per day summed across the follow-up period for each patient.

${ }^{c}$ Costs discounted at $3 \%$ per annum.

${ }^{d}$ Costs discounted at $3.5 \%$ per annum.

${ }^{\text {e }}$ Rate ratio, $95 \% \mathrm{Cls}$, and $P$-value estimated using Stata's MEGLM command.

than those in the placebo arm, yet means ratios were consistent with higher costs. These inconsistencies can be attributed to the resampling-with-replacement procedure employed with the standard approach to bootstrapping, whereas the modeling procedure accounts for correlations across patients enrolled within a country and adjusts for patient-level differences in duration of follow-up.

More compelling evidence is that all-cause hospitalization rates began to diverge in the second year of follow-up and continued through the fifth year. Evidence from previous long-term trials in diabetes suggests that changes in $\mathrm{HbA} 1 \mathrm{c}$ require long follow-up periods to observe cardiovascular benefits, and it has been hypothesized that a "metabolic memory" may contribute to such effects. ${ }^{20,21}$ Post-hoc analyses suggest that hospitalization rates were approximately $10 \%$ lower among patients treated with sitagliptin. The net clinical and economic impact is dependent on the absolute hospitalization rate, which was 0.24 per patient-year for the placebo group, but varied widely across countries, ranging (in countries with $\geq 100$ patients per arm) from 0.06 per patient-year in India to 0.57 per patient-year in New Zealand. The hospitalization rate for the placebo arm in the USA, where more than 1000 patients were randomized to placebo, was 0.34 per patient-year. At 10000 USD per hospitalization, a 10\% reduction in hospitalizations would result in savings of 335 USD per patient-year in the USA. By comparison, the annual cost of sitagliptin, based on the WAC, was estimated at approximately 3300 USD. Discounts and rebates negotiated by payers are not transparent, but a recent report from the US Office of the Inspector General reports a mean rebate of $23.1 \%$ of the average manufacturer's price for brand-name drugs paid for by Medicaid. ${ }^{22}$ With this discount, treatment costs of approximately 2500 USD would be marginally offset with lower inpatient costs. In other jurisdictions, with higher rates of hospital admission and/or larger discounts and rebates for sitagliptin, the offset could be larger. In the UK cost analysis, lower annual costs of sitagliptin treatment (relative to the USA), combined with savings in hospitalization costs (relative to the placebo group), reduced total incremental costs to approximately 300 GBP per patient-year.

The present study is a resource utilization study conducted within a clinical trial and cannot be compared directly with costeffectiveness analyses of sitagliptin, which typically estimate incremental lifetime costs and quality-adjusted life-years (QALYs) as the outcome measure. Previously published cost-effectiveness analyses have suggested that, when sitagliptin is compared with sulfonylureas in patients receiving metformin monotherapy, lifetime gains with use of sitagliptin range from 0.04 to 0.1 QALYs. ${ }^{23}$ Using the upper end of that range, incremental lifetime costs with use of sitagliptin less than 10000 USD would maintain an incremental costeffectiveness ratio of $<100000$ USD per QALY. In a UK setting, lifetime costs with use of sitagliptin would have to be less than 2000 GBP to maintain an incremental cost-effectiveness ratio of $<20000$ GBP per QALY. 
TABLE 2 Impact of sitagliptin on all-cause hospitalizations by subgroup

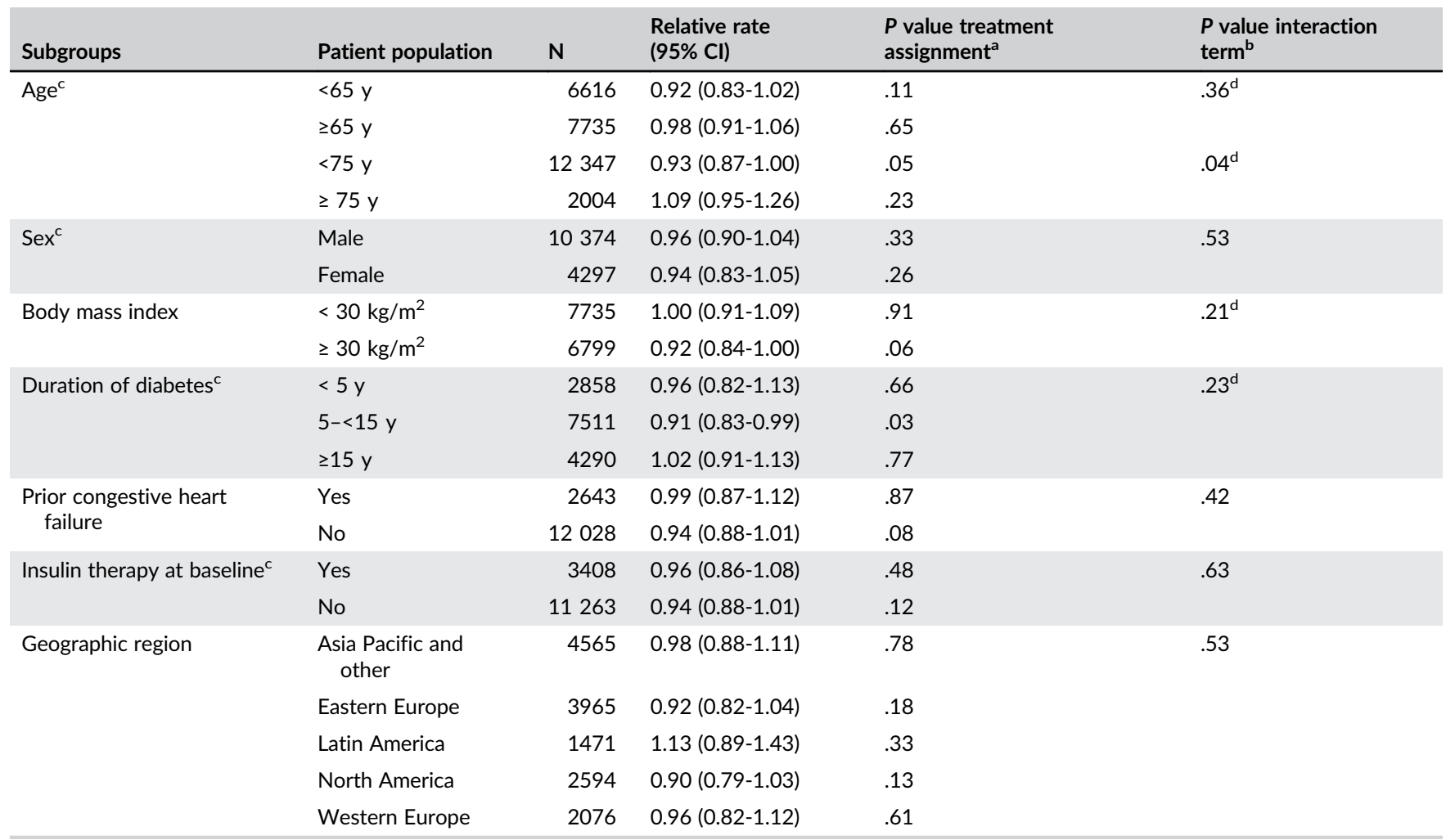

a $P$ values for treatment assignment for each subgroup.

${ }^{b} P$ values for interactions between treatment arm and categorical subgroup.

c Prespecified subgroups in trial protocol.

${ }^{\mathrm{d}} P$ values for interaction terms for interactions between treatment group and continuous, linear variables representing are .32 for age, .07 for body mass index, and .18 for duration of diabetes.

Our findings raise some methodological issues associated with conducting economic evaluations using data from multinational clinical trials. First, assigning costs to medical resource use in such trials is complex. ${ }^{24-26}$ We applied a pragmatic approach, assigning US-based costs to medical resources used by patients from all countries, but adjusting inpatient length of stay to account for variations in practice patterns. We also applied UK costs in a sensitivity analysis to evaluate the impact of different price weights on the results. However, all approaches must recognize that practice patterns, and thus rates of medical resource use, can vary dramatically across jurisdictions. We also realize that the constraints of excluding GLP-1 receptor agonists and other DPP-4 inhibitors from usual care means that the results may be less generalizable to countries where these drugs are treatment options.

The TECOS trial provides valuable large-scale pragmatic insights into the short- to mid-term incremental effects on medical resource use and costs when sitagliptin is added to usual care for type 2 diabetes patients with cardiovascular disease. We observed small reductions in insulin use and hospitalization rates when sitagliptin was added to usual care. These cost-savings slightly offset sitagliptin treatment costs over a 3-year period. The amount of drug cost offset in the USA would be proportionately larger, with greater discounts and rebates on the WAC for sitagliptin. As type 2 diabetes is a chronic, progressive and complex disease, further research is needed

TABLE 3 Hospitalization rates and relative impact of sitagliptin on all-cause hospitalizations by minimum follow-up duration

\begin{tabular}{|c|c|c|c|c|c|c|}
\hline \multirow[b]{2}{*}{ Duration of follow-up (y) } & \multirow[b]{2}{*}{$\mathbf{N}$} & \multicolumn{3}{|c|}{ Unadjusted $^{a}$} & \multirow{2}{*}{$\begin{array}{l}\text { Adjusted }^{\mathrm{b}} \\
\text { Relative rate }\end{array}$} & \multirow[b]{2}{*}{$P$ value $^{b}$} \\
\hline & & Sitagliptin & Placebo & Relative rate & & \\
\hline$\geq 2$ & 13700 & 0.203 & 0.223 & 0.91 & $0.94(0.88-1.01)$ & .07 \\
\hline$\geq 2.5$ & 9674 & 0.200 & 0.227 & 0.88 & $0.90(0.83-0.97)$ & .01 \\
\hline$\geq 3$ & 7189 & 0.199 & 0.225 & 0.88 & $0.90(0.83-0.98)$ & .02 \\
\hline$\geq 3.5$ & 4440 & 0.183 & 0.213 & 0.86 & $0.89(0.79-0.99)$ & .03 \\
\hline$\geq 4$ & 2641 & 0.186 & 0.215 & 0.86 & $0.89(0.77-1.02)$ & .10 \\
\hline
\end{tabular}

${ }^{a}$ Number of hospitalizations divided by patient-years of follow-up in each time interval.

${ }^{\mathrm{b}}$ Adjusted relative rate and $P$ values from SAS GLIMMIX procedure representing relative impact of sitagliptin vs placebo, accounting for country-level correlation and adjusted for age, sex, prior CV disease and years since diabetes diagnosis. 
concerning the impact of DPP-4 inhibitor treatment on clinical outcomes, health care resource utilization and costs in broader diabetes populations such as those with higher HbA1c levels, with and without pre-existing cardiovascular disease.

\section{ACKNOWLEDGMENTS}

R. R. H. is a National Institute for Health Research Senior Investigator.

The authors thank Jyotsna Garg, MS from the Duke Clinical Research Institute for her assistance with data access and verifying variable definitions and coding; Felicia Graham, MBA for her contributions to data monitoring and project management; and John R. Cook, PhD and Jieling Chen, PhD for their early contributions in planning for the economic evaluation.

Parts of this study were presented in abstract form at the 77th Scientific Sessions of the American Diabetes Association, San Diego, CA, June 9-13, 2017, and at the 53rd EASD Annual Meeting, Lisbon, Portugal, September 11-15, 2017.

\section{Conflict of interest}

S. D. R. has made available online a detailed listing of financial disclosures (http://www.dcri.duke.edu/about-us/conflict-of-interest/). J. B. B. has received consulting fees from PhaseBio and research support from AstraZeneca, Bayer, Boehringer-Ingelheim, BristolMyers Squibb, Eli Lilly, GI Dynamics, GlaxoSmithKline, Intarcia Therapeutics, J\&J, Lexicon, Medtronic Minimed, National Institutes of Health, Novo Nordisk, Orexigen, Sanofi, Scion NeuroStim, Takeda and Theracos; owns stocks/shares in PhaseBio; and has served as an advisor under contract with his employer for AstraZeneca, Dance Biopharm, Eli Lilly, Elcelyx, GI Dynamics, Lexicon, Merck, Metavention, Novo Nordisk, Orexigen and VTv Therapeutics; and has received other support from Adocia, Insulin Algorithms, Dexcom, Fractyl, NovaTarg and Shenzen HighTide. J. B. G. has received grants from Merck, AstraZeneca and GlaxoSmithKline, grants and personal fees from Merck, other support from Boehringer-Ingelheim, and personal fees from Bioscientifica and The Endocrine Society. K. D. K. is an employee of Merck \& Co., Inc., the manufacturer of sitagliptin, and owns stock and stock options in Merck \& Co., Inc. L. R. is an employee of Merck \& Co., Inc., the manufacturer of sitagliptin, and owns stock and stock options in Merck \& Co., Inc. F. V. $W$. has received study grants and personal fees from Merck, AstraZeneca and Boehringer-Ingelheim. E. D. P. has received grants and personal fees from Janssen, grants from Eli Lilly and personal fees from AstraZeneca, Bayer and Sanofi. R. R. H. has received grants from AstraZeneca during the conduct of the study; and grants and personal fees from Bayer, Boehringer-Ingelheim and Merck, personal fees from Novartis, Amgen and Servier; and other support from Elcelyx, GlaxoSmithKline, Janssen and Takeda outside the submitted work. The other authors report no potential conflicts of interest relevant to this article.

\section{Author contributions}

S. D. R. contributed to plans to collect medical resource use data in the trial, wrote the costing and data analysis plan, advised on US cost assignment, and wrote the manuscript. Y. L. led the collection of unit costs, and performed US and UK cost assignment and statistical analysis of the data. J. L. provided UK unit costs and advised on UK cost assignment, performed statistical analysis, and reviewed/edited the manuscript. L. R. reviewed and advised on the costing and data analysis plan, and reviewed/edited the manuscript. J. A. assisted with assigning DRG and Current Procedural Terminology codes to support cost assignment, and reviewed and edited the manuscript. A. A., J. B. B., J. B. G., K. D. K., A. R., F. V. W. and E. D. P. reviewed/edited the manuscript. A. M. G. contributed to plans to collect medical resource use data in the trial, wrote the costing and data analysis plan, advised on UK cost assignment, and wrote the manuscript. R. R. H. designed and ran the TECOS trial, contributed to plans to collect medical resource use data in the trial, and reviewed/edited the manuscript. S. D. R. is the guarantor of this work and, as such, had full access to all the data in the study and takes responsibility for the integrity of the data and the accuracy of the data analysis.

\section{ORCID}

Shelby D. Reed (D) http://orcid.org/0000-0002-7654-4464

Rury R. Holman (D) http://orcid.org/0000-0002-1256-874X

\section{REFERENCES}

1. GBD 2015 DALYs and HALE Collaborators. Global, regional, and national disability-adjusted life-years (DALYs) for 315 diseases and injuries and healthy life expectancy (HALE), 1990-2015: a systematic analysis for the Global Burden of Disease Study 2015. Lancet. 2016; 388:1603-1658.

2. da Rocha Fernandes J, Ogurtsova K, Linnenkamp U, et al. IDF Diabetes Atlas estimates of 2014 global health expenditures on diabetes. Diabetes Res Clin Pract. 2016;117:48-54.

3. Ogurtsova K, da Rocha Fernandes JD, Huang Y, et al. IDF Diabetes Atlas: global estimates for the prevalence of diabetes for 2015 and 2040. Diabetes Res Clin Pract. 2017;128:40-50.

4. The Emerging Risk Factors Collaboration. Diabetes mellitus, fasting blood glucose concentration, and risk of vascular disease: a collaborative meta-analysis of 102 prospective studies. Lancet. 2010;375:2215-2222.

5. Rawshani A, Rawshani A, Franzén $S$, et al. Mortality and cardiovascular disease in type 1 and type 2 diabetes. N Engl J Med. 2017;376: 1407-1418.

6. Emerging Risk Factors Collaboration. Diabetes mellitus, fasting glucose, and risk of cause-specific death. N Engl J Med. 2011;364:829-841.

7. Nissen SE, Wolski K. Effect of rosiglitazone on the risk of myocardial infarction and death from cardiovascular causes. N Engl J Med. 2007; 356:2457-2471.

8. Rao AD, Kuhadiya N, Reynolds K, Fonseca VA. Is the combination of sulfonylureas and metformin associated with an increased risk of cardiovascular disease or all-cause mortality?: a meta-analysis of observational studies. Diabetes Care. 2008;31:1672-1678.

9. US Department of Health and Human Services, Food and Drug Administration, Center for Drug Evaluation and Research. Guidance for industry: Diabetes mellitus - evaluating cardiovascular risk in new antidiabetic therapies to treat type 2 diabetes; 2008. http://www.fda. gov/downloads/Drugs/GuidanceComplianceRegulatorylnformation/ Guidances/ucm071627.pdf. Accessed February 6, 2017.

10. European Medicine Agency, Committee for Medicinal Products for Human Use. Guideline on clinical investigation of medicinal products in the treatment of diabetes mellitus; 2010. http://www.ema.europa. 
eu/docs/en_GB/document_library/Scientific_guideline/2010/02/ WC500073570.pdf. Accessed January 25, 2017.

11. Green JB, Bethel MA, Paul SK, et al. Rationale, design, and organization of a randomized, controlled Trial Evaluating Cardiovascular Outcomes with Sitagliptin (TECOS) in patients with type 2 diabetes and established cardiovascular disease. Am Heart J. 2013;166:983-989.

12. Green JB, Bethel MA, Armstrong PW, et al. Effect of sitagliptin on cardiovascular outcomes in type 2 diabetes. N Engl J Med. 2015;373: 232-242.

13. Reed SD, Kaul P, Li Y, et al. Medical resource use, costs and quality of life in acute decompensated heart failure: findings from ASCEND-HF. J Card Fail. 2013;19:611-620.

14. Centers for Medicare and Medicaid Services. IPPS final rule home page;FY 2015. https://www.cms.gov/Medicare/Medicare-Fee-forService-Payment/AcutelnpatientPPS/FY2015-IPPS-Final-Rule-HomePage.html. Accessed June 1, 2015.

15. Centers for Medicare and Medicaid Services. National physician fee schedule payment amount file. http://www.cms.hhs.gov/Physician FeeSched/01_overview.asp. Accessed May 1, 2015.

16. First Databank. Policies: Drug pricing policy. http://www.fdbhealth. com/policies/drug-pricing-policy/ Accessed June 1, 2017.

17. Dong H, Robison LL, Leisenring WM, Martin LJ, Armstrong GT, Yasui Y. Estimating the burden of recurrent events in the presence of competing risks: the method of mean cumulative count. Am J Epidemiol. 2015;181:532-540.

18. Efron B, Tibshirani RJ. Bootstrap methods for standard errors, confidence intervals, and other measures of statistical accuracy. Stat Sci. 1986;1:54-77.

19. McGuire DK, Van de Werf F, Armstrong PW, et al. Association between sitagliptin use and heart failure hospitalization and related outcomes in type 2 diabetes mellitus: secondary analysis of a randomized clinical trial. JAMA Cardiol. 2016;1:126-135.

20. Holman RR, Paul SK, Bethel MA, Matthews DR, Neil HA. 10-year follow-up of intensive glucose control in T2D. N Engl J Med. 2008; 359:1577-1589.
21. Ceriello A, Ihnat MA, Thorpe JE. Clinical review 2: the "metabolic memory": is more than just tight glucose control necessary to prevent diabetic complications? J Clin Endocrinol Metab. 2009;94:410-441.

22. Department of Health and Human Services, Office of Inspector General. Medicaid rebates for brand-name drugs exceeded part $D$ rebates by a substantial margin. April 2015. OEl-03-13-00650. https://oig. hhs.gov/oei/reports/oei-03-13-00650.pdf. Accessed June 7, 2017.

23. Schwarz B, Gouveia M, Chen J, et al. Cost-effectiveness of sitagliptin-based treatment regimens in European patients with type 2 diabetes and haemoglobin A1c above target on metformin monotherapy. Diabetes Obes Metab. 2008;10(suppl 1):43-55.

24. Reed SD, Anstrom KJ, Bakhai A, et al. Conducting economic evaluations alongside multinational clinical trials: toward a research consensus. Am Heart J. 2005;149:434-443.

25. Glick HA, Doshi JA, Sonnad SS, Polsky D. Economic Evaluation in Clinical Trials. New York, NY: Oxford University Press; 2007.

26. Raikou M, Briggs A, Gray A, McGuire A. Centre-specific or average unit costs in multi-Centre studies? Some theory and simulation. Health Econ. 2000;9:191-198.

\section{SUPPORTING INFORMATION}

Additional Supporting Information may be found online in the supporting information tab for this article. 